\title{
STRONG BANACH-SAKS OPERATORS
}

\author{
MOHAMED HAJJI
}

\begin{abstract}
In this paper, we introduce a new class of operators, called strong Banach-Saks operators, related to the Banach-Saks and L-weakly compact operators. We first prove that every strong Banach-Saks operator from a Banach space $Z$ into a Banach lattice $F$ is Banach-Saks. Then we show that if $F$ is order continuous, the notions of strong Banach-Saks and Banach-Saks operators coincide. Finally, we close this paper by a new characterization of order continuous Banach lattices.

Вводиться новий клас операторів, так звані сильні оператори Банаха-Сакса, пов'язані з операторами Банаха-Сакса i L-слабко компактними операторами. Доведено, що кожен сильний оператор Банаха-Сакса з банахового простору $Z$ у банахову решітку $F$ є оператором Банаха-Сакса. Далі, якщо $F \in$ порядково неперервним, то властивості оператора Банаха-Сакса і сильного оператора БанахаСакса співпадають. Нарешті, в статті дано нову характеризацію порядково неперервних банахових решіток.
\end{abstract}

\section{INTRODUCTION}

In [3], S. Banach and S. Saks showed that for $1<p<\infty$, every bounded sequence in $L_{p}[0,1]$ has a subsequence $\left(y_{n}\right)$ whose arithmetic means converge in norm. That is

$$
\frac{1}{n} \sum_{k=1}^{n} y_{k} \stackrel{\|\cdot\|_{p}}{\longrightarrow} x
$$

This prompted A. Brunel and L. Sucheston [6] to qualify every Banach space with this property as a Banach-Saks space. Every Banach-Saks space is reflexive, see 11, Proposition 2.3]. The converse statement is not true in general. That is, there are reflexive spaces without the Banach-Saks property [2]. Inspired by the preceding papers, B. Beauzamy introduced in [4 the notion of a Banach-Saks operator. We say that an operator $T: X \longrightarrow Y$ between two Banach spaces is a Banach-Saks operator if $T$ maps the closed unit ball $B_{X}$ of $X$ onto a Banach-Saks subset of $Y$. A bounded subset $A$ of $X$ is said to be Banach-Saks if each sequence $\left(x_{n}\right)$ in $A$ has a subsequence $\left(y_{n}\right)$ whose arithmetic means converge in norm. Observe that a compact operator must be a Banach-Saks operator. The two notions coincide when $Y$ has the Schur property. Every Banach-Saks operator is weakly compact. If $Y$ has the positive Schur property, then weakly compact and Banach-Saks operators coincide.

The class of L-weakly compact operators was introduced by Meyer-Nieberg[8]. Recall that a bounded subset $A$ of a Banach lattice $E$ is said to be L-weakly compact, if $\left\|x_{n}\right\| \longrightarrow 0$ for every disjoint sequence $\left(x_{n}\right)_{n}$ in the solid hull of $A$. A linear operator $T$ from a Banach space $X$ into a Banach lattice $F$ is said to be L-weakly compact if so is $T\left(B_{X}\right)$. Note that (by Proposition 3.6.5 in [9]) every L-weakly compact operator is weakly compact.

In this paper, we introduce a new class of operators, called strong Banach-Saks operators, related to the Banach-Saks and L-weakly compact operators. We first prove that every strong Banach-Saks operator from a Banach space $Z$ into a Banach lattice

2020 Mathematics Subject Classification. 46B42, 47B60, 47B65.

Keywords. Banach-Saks; Banach lattice; L-weakly compact; order continuous norm. 
$F$ is Banach-Saks. We also show that if $F$ is order continuous, the notion of strong Banach-Saks and Banach-Saks operators coincide. Finally, we close this paper by a new characterization of order continuous Banach lattices.

Our terminology and notations are standard, and we refer to [1] and [9] for unexplained definitions and properties about Banach lattices and operators on them.

\section{Strong Banach-Saks Operators}

We start by the following definition.

Definition 2.1. A linear operator $T$ from a Banach space $X$ into a Banach lattice $F$ is said to be strong Banach-Saks if for every bounded sequence $\left(x_{n}\right)_{n}$ in $X$, the sequence of images $\left(T x_{n}\right)_{n}$ has a subsequence which is Cesàro L-weakly compact in $F$ (i.e, there exists a subsequence $\left(T y_{k}\right)_{k}$ such that $\left\{\frac{1}{n} \sum_{l=1}^{n} T y_{l}, n \in \mathbb{N}\right\}$ is an L-weakly compact subset of $F$ ).

To continue our discussion, we need the next Lemma.

Lemma 2.2. [5, Lemma 2.4] For every nonempty bounded subset $A \subset E$, the following assertions are equivalent.

(1) A is L-weakly compact.

(2) $f_{n}\left(x_{n}\right) \rightarrow 0$ for every sequence $\left(x_{n}\right)$ of $A$ and every disjoint sequence $\left(f_{n}\right)$ of $B_{E^{\prime}}$.

Note that every L-weakly compact operator $T: X \rightarrow F$ is strong Banach-Saks. Indeed, let $\left(x_{n}\right)_{n}$ be a bounded sequence of $X$. Since $T$ is L-weakly compact, it follows from Lemma 2.2 that $f_{n}\left(T x_{n}\right) \rightarrow 0$ for every disjoint sequence $\left(f_{n}\right)$ of $B_{F^{\prime}}$. Thus, $\frac{1}{n} \sum_{k=1}^{n} f_{k}\left(T x_{k}\right) \rightarrow 0$. This shows that $T$ is strong Banach-Saks. Every strong BanachSaks operator is a weakly compact operator. The details follow.

Theorem 2.3. Every strong Banach-Saks operator $T$ from a Banach space $Z$ into a Banach lattice $F$ is weakly compact.

Proof. Let $\left(x_{n}\right)$ be a bounded sequence in $Z$. Since $T$ is strong Banach-Saks, it follows that there exists a subsequence $\left(y_{n}\right)_{n}$ of $\left(x_{n}\right)_{n}$ such that $\left\{\frac{1}{n} \sum_{k=1}^{n} T y_{k} ; \quad n \in \mathbb{N}\right\}$ is L-weakly compact subset of $F$. On the other hand, note that the sequence $\left(e_{n}\right)_{n}$ is not Banach-Saks, where $\left(e_{n}\right)$ is the standard basis of $l_{1}$. Now, an easy application of Theorem 4.32 in [1] shows that $\left\{\frac{1}{n} \sum_{k=1}^{n} e_{k} ; n \in \mathbb{N}\right\}$ is not relatively weakly compact, in particular, $\left\{\frac{1}{n} \sum_{k=1}^{n} e_{k} ; \quad n \in \mathbb{N}\right\}$ is not L-weakly compact (see Proposition 3.6.5 in [9] ). Hence, by the Rosenthal's $l_{1}$ Theorem, there exists a subsequence $\left(z_{n}\right)$ of $\left(y_{n}\right)$ such that $\left(T z_{n}\right)$ is weak Cauchy. According to Theorem 9.3.1 in [7, there exists some $z^{\prime \prime} \in F^{\prime \prime}$ so that $T z_{n} \stackrel{\sigma\left(F^{\prime \prime}, F^{\prime}\right)}{\longrightarrow} z^{\prime \prime}$. Now, since $\left\{\frac{1}{n} \sum_{k=1}^{n} T z_{k} ; \quad n \in \mathbb{N}\right\}$ is L-weakly compact, it follows from Proposition 3.6.5 in [9] that there is a subsequence $\left(t_{n}\right)_{n}$ of $\left(z_{n}\right)_{n}$ such that $\frac{1}{n} \sum_{k=1}^{n} T t_{k} \stackrel{\sigma\left(F, F^{\prime}\right)}{\longrightarrow} z \in F$. So $z=z^{\prime \prime}$, and consequently $\left(T\left(t_{n}\right)\right)_{n}$ converges weakly to $z \in F$.

Recall that a Banach lattice $E$ is said to be order continuous if $\lim _{\alpha}\left\|x_{\alpha}\right\|=0$ for every decreasing net $\left(x_{\alpha}\right)_{\alpha}$ in $E$ such that $\wedge_{\alpha} x_{\alpha}=0$. Let $E$ be a Banach lattice. An element $e \in E$ is called weak unit if for $h \in E, e \wedge h=0$ implies $h=0$.

If $E$ is an order continuous Banach lattice with weak unit, then there exists a probability space $(\Omega, \Sigma, \mu)$, an order ideal $I$ of $L_{1}(\Omega, \Sigma, \mu)$, a lattice norm $\|\cdot\|_{I}$ on $I$, and an order isometry $j$ from $E$ onto $\left(I,\|\|_{I}\right)$ such that the canonical inclusion from $I$ into $L_{1}(\Omega, \Sigma, \mu)$ is continuous with $\|f\|_{1} \leq\|f\|_{I}$ (see Theorem 1.b.14 in [14). This implies that $j$ is continuous as an operator from $E$ into $L_{1}(\Omega, \Sigma, \mu)$. Note that a separable subspace $X$ of an order continuous Banach lattice $E$ is included in some closed order ideal $Y$ of $E$ with weak unit (see Proposition 1.a.9 in [14]). Thus, $\overline{F_{X}}$ ( $F_{X}$ the ideal generated 
by $X$ ) has a weak unit. In terms of order continuous Banach lattices, the convergence of a bounded sequence is characterized as follows.

Lemma 2.4. Let $E$ be a Banach lattice with order continuous norm, and $\left(g_{n}\right)_{n}$ be a bounded sequence in $E\left(\overline{E_{\left[g_{n}\right]}}\right.$ representable as an order ideal in $L_{1}(\Omega, \Sigma, \mu)$ for some probability measure $\mu)$. Then:

$\left(g_{n}\right)_{n}$ is convergent in $E$ if and only if $\left(g_{n}\right)_{n}$ is L-weakly compact and $\|\cdot\|_{1}$-convergent.

Proof. Since $\left[g_{n}\right]$ is a separable subspace of $E$, it follows from Proposition 1.a.9 in 14] that $E_{1}=\overline{E_{\left[g_{n}\right]}}\left(E_{\left[g_{n}\right]}\right.$ the ideal generated by $\left.\left(g_{n}\right)_{n}\right)$ is a Banach lattice with weak unit. Thus, from Lemma 1.4.2 in [12] it easily follows that $\left(g_{n}\right)_{n}$ is convergent in $E_{1}$ if and only if $\left(g_{n}\right)$ is L-weakly compact and $\|\cdot\|_{1}$-convergent, which finishes the proof.

A Banach space $E$ has the weak Banach-Saks property (or it is weakly Banach-Saks) if every weakly convergent sequence $\left(x_{n}\right)_{n}$ in $E$ has a subsequence which is Cesàro convergent.

Theorem 2.5. (Szlenk [16]) Let $(\Omega, \Sigma, \mu)$ be a probability space. Then $L_{1}(\Omega, \Sigma, \mu)$ is weakly Banach-Saks.

Our next goal is to establish that a strong Banach-Saks operator is a Banach-Saks operator. To do this, we need the following Lemma. That is the general version of Theorem 5.66 in [1].

Lemma 2.6. Let $A$ be an L-weakly compact subset of $E$. If $E_{A}$ is the ideal generated by $A$, then $\overline{E_{A}}$ is a Banach lattice with order continuous norm.

Proof. Let $E_{A}$ be the ideal generated by $A$ in $E$. By Theorems 4.13 and 4.11 in [1, it suffices to show that every order bounded disjoint sequence in $E_{A}$ is norm convergent to zero. Let $\left(y_{n}\right)_{n}$ be a disjoint sequence with $0 \leq y_{n} \leq y$ for all $n$ and some $y \in E_{A}$. Then there exist $x_{1}, \ldots, x_{n_{0}} \in A_{+}$and $\lambda>0$ such that

$$
y \leq \lambda \sum_{i=1}^{n_{0}} x_{i} .
$$

From the Riesz decomposition property(see Theorem 1.13 in [1]), there exist $y_{1}^{n}, \ldots, y_{n_{0}}^{n}$ in $E_{+}$with

$$
y_{n}=y_{1}^{n}+\ldots+y_{n_{0}}^{n}, \text { and } y_{i}^{n} \leq \lambda x_{i}
$$

for all $n \in \mathbb{N}$ and $i \in\left\{1, \ldots, n_{0}\right\}$. Clearly, for each $i$ the sequence $\left(y_{i}^{n}\right)_{n}$ is disjoint and included in Sol $A$. The L-weak compactness of $A$ guarantees that $y_{n} \rightarrow 0$ in norm. So $\overline{E_{A}}$ is order continuous.

Let $X$ be a Banach space. A sequence $\left(x_{n}\right)$ in $X$ is said to be Cesàro convergent if its Cesàro means converge in norm. An operator $T$ from a Banach space $X$ to a Banach space $Y$ is called a Banach-Saks operator if for any norm bounded sequence $\left(x_{n}\right)$ in $X$, $\left(T x_{n}\right)_{n}$ has a Cesàro convergent subsequence.

Theorem 2.7. Every strong Banach-Saks operator $T$ from a Banach space $Z$ into a Banach lattice $F$ is Banach-Saks.

Proof. Let $\left(x_{n}\right)_{n}$ be a bounded sequence in $Z$. Since $T$ is strong Banach-Saks, it follows that there exists a subsequence $\left(y_{n}\right)_{n}$ of $\left(x_{n}\right)_{n}$ such that $\left\{\frac{1}{n} \sum_{k=1}^{n} T y_{k} ; \quad n \in \mathbb{N}\right\}$ is Lweakly compact subset in $F$. Let $A=\left\{\frac{1}{n} \sum_{k=1}^{n} T y_{k} ; \quad n \in \mathbb{N}\right\}$. Then from Lemma 2.6 we see that $\overline{F_{A}}\left(F_{A}\right.$ is the ideal generated by $\left.A\right)$ is order continuous.

Now, since $X:=\left[T y_{n}\right]$ is a separable subspace of $F_{A}$, it follows from Proposition 1.a.9 in [14] that $\overline{F_{X}}$ is an order ideal with a weak order unit and so can be represented as 
a dense order ideal of $L_{1}(\Omega, \Sigma, \mu)$ for some probability measure $\mu$, such that the formal inclusion

$$
j: \overline{F_{X}} \hookrightarrow L_{1}(\Omega, \Sigma, \mu)
$$

is continuous ([14, Theorem 1.b.14). By Theorem 2.3 the sequence $\left(y_{n}\right)$ has a subsequence $\left(z_{n}\right)$ such that $\left(T z_{n}\right)$ converges weakly to some $z \in \overline{F_{X}}$. Thus, $j\left(T z_{n}\right)$ converges weakly in $L_{1}(\Omega, \Sigma, \mu)$. The fact that $L_{1}(\Omega, \Sigma, \mu)$ is weakly Banach-Saks(by Theorem 2.5), and hence without loss of generality we can assume that there is some $f \in L_{1}(\Omega, \Sigma, \mu)$ such that:

$$
\left\|j\left(\frac{1}{n} \sum_{k=1}^{n} T z_{k}\right)-f\right\|_{1} \longrightarrow 0 .
$$

Since $\left\{\frac{1}{n} \sum_{k=1}^{n} T z_{k} ; n \in \mathbb{N}\right\}$ is L-weakly compact subset in $F_{X}$, it follows from Lemma 2.4 that $\frac{1}{n} \sum_{k=1}^{n} T z_{k}$ converges in $F$.

It is interesting to know that the converse of the preceding theorem is in general false, as shown in the following.

Example 2.8. Consider the linear operator $T: l_{1} \rightarrow l_{\infty}$ defined by

$$
T\left(x_{1}, x_{2}, \ldots\right)=\left(\sum_{i=1}^{\infty} x_{i}, \sum_{i=1}^{\infty} x_{i}, \ldots\right)=\sum_{i=1}^{\infty} x_{i}(1,1, \ldots) .
$$

Evidently, $T$ is Banach-Saks (it has rank one). Let $\left(e_{n}\right)$ be the sequence of standard unit vectors in $l_{1}$. Then $\left(e_{n}\right)$ is a disjoint sequence in the solid hull of $\left\{\frac{1}{n} \sum_{k=1}^{n} T e_{k}, n \in \mathbb{N}\right\}$ and $\left\|e_{n}\right\|_{\infty}=1$. Consequently the operator $T$ fails to be strong Banach-Saks.

However, it holds for order continuous Banach lattices, as follows from the next theorem:

Theorem 2.9. A bounded linear operator from a Banach space $Z$ into an order continuous Banach lattice $F$ is Banach-Saks if and only if it is strong Banach-Saks.

Proof. From Theorem 2.7, it is enough to show that every Banach-Saks operator from $Z$ into $F$ is strong Banach-Saks. To this end, let $\left(x_{n}\right)$ be a bounded sequence in $Z$. Since $T$ is Banach-Saks, it follows that there is a subsequence $\left(z_{n}\right)$ of $\left(x_{n}\right)$ such that for every subsequence $\left(y_{n}\right)$ of $\left(z_{n}\right)$, we have that $T\left(y_{n}\right)$ is Cesàro convergent to some $y \in F$, that is:

$$
\left\|\frac{1}{n} \sum_{k=1}^{n} T y_{k}-y\right\| \rightarrow 0
$$

Let $\left(w_{k}\right)_{k}$ be a disjoint sequence in the solid hull of $\left\{\frac{1}{n} \sum_{k=1}^{n} T y_{k} ; n \in \mathbb{N}\right\}$. Without loss of generality we can assume that $\left|w_{n}\right| \leq\left|\frac{1}{n} \sum_{k=1}^{n} T y_{k}\right|$ holds for all $n \in \mathbb{N}$. Then

$$
\left|w_{n}\right| \leq\left|\frac{1}{n} \sum_{k=1}^{n} T y_{k}-y\right|+|y|
$$

From the Riesz decomposition property (see [1, Theorem 1.13]), it follows that for each $n$ there exist $w_{n}^{1}, w_{n}^{2} \in F_{+}$such that $\left|w_{n}\right|=w_{n}^{1}+w_{n}^{2}$ with:

$$
w_{n}^{1} \leq\left|\frac{1}{n} \sum_{k=1}^{n} T y_{k}-y\right| \quad \text { and } \quad w_{n}^{2} \leq|y| .
$$

By 2.1 the sequence $\left(w_{n}^{1}\right)_{n}$ converges to 0 in $F$. On the other hand, since $\left(w_{n}^{2}\right)_{n}$ is order bounded and disjoint $\left(0 \leq w_{n}^{2} \leq\left|w_{n}\right|\right)$, it follows from Theorem 4.14 in [1] that $\left(w_{n}^{2}\right)_{n}$ converges to 0 in $F$. Thus $\lim _{n}\left\|w_{n}\right\|=0$, and so $T$ is strong Banach-Saks. 
Recall that a subset $A$ of a Banach space $X$ is called Banach-Saks if every sequence in $A$ has a Cesàro convergent subsequence. A Banach space $X$ is said to have the BanachSaks property when its unit ball is a Banach-Saks set.

Corollary 2.10. For a Banach lattice $E$ the following statements are equivalent.

(1) E has the Banach-Saks property.

(2) The identity operator $I: E \rightarrow E$ is strong Banach-Saks.

Proof. First, assume that $E$ has the Banach-Saks property. From Corollary of Theorem 1 in [10] we know that $E$ is reflexive, in particular it is order continuous (see Theorem 4.70 in [1]). Since the identity operator $I: E \rightarrow E$ is Banach-Saks, it follows from Theorem 2.9 that $I: E \rightarrow E$ is strong Banach-Saks.

For the converse assume that $I: E \rightarrow E$ is strong Banach-Saks, it follows from Theorem 2.7 that $E$ is Banach-Saks.

A Banach lattice $E$ has the positive Schur property if weakly null sequences with positive terms are norm null.

Corollary 2.11. For a bounded linear operator $T$ from a Banach space $Z$ into a Banach lattice $F$ with positive Schur property the following statements are equivalent.

(1) $T$ is L-weakly compact.

(2) $T$ is strong Banach-Saks.

(3) $T$ is Banach-Saks.

(4) $T$ is weakly compact.

Proof. (1) $\Longrightarrow(2)$. Let $\left(x_{n}\right)_{n}$ be a bounded sequence of $Z$. By Theorem 5.61 in [1] the sequence $T\left(x_{n}\right)$ has a weakly convergent subsequence in $F$ (which we shall denote by $T\left(x_{n}\right)$ again). Therefore, the sequence $\left(\frac{1}{n} \sum_{k=1}^{n} T x_{k}\right)_{n}$ also has a weakly convergent subsequence in $F$. Next, let $\left(w_{n}\right)$ be a disjoint sequence in the solid hull of $\left(\frac{1}{n} \sum_{k=1}^{n} T x_{k}\right)_{n}$, then $\left(\left|w_{n}\right|\right)$ is also in the solid hull of $\left(\frac{1}{n} \sum_{k=1}^{n} T x_{k}\right)_{n}$. Now an easy application of Theorem 4.34 shows that $\left(\left|w_{n}\right|\right)$ converges weakly to zero. Since $F$ has the positive Schur property, it follows that $\lim _{n}\left\|\left|w_{n}\right|\right\|=\lim _{n}\left\|w_{n}\right\|=0$. Consequently, $\left(\frac{1}{n} \sum_{k=1}^{n} T x_{k}\right)_{n}$ is L-weakly compact subset of $F$, and so $T$ is strong Banach-Saks.

$(2) \Longrightarrow(3)$. It is a direct application of Theorem 2.7

$(3) \Longrightarrow(4)$. It follows from Proposition 2.3 in [11.

$(4) \Longrightarrow(1)$. Since $F$ has the positive Schur property and $T\left(B_{Z}\right)$ is relatively weakly compact subset of $F$, it follows from Theorem 4.34 in [1] that $T$ is L-weakly compact.

The domination property for strong Banach-Saks operators is the following.

Theorem 2.12. Let $E$ and $F$ be Banach lattices. If $0 \leq S \leq T: E \longrightarrow F$ with $T$ is strong Banach-Saks, then $S$ is also strong Banach-Saks.

Proof. Suppose that $T$ is strong Banach-Saks and let $\left(x_{n}\right)$ be a sequence in $E$. Then there exists a subsequence $\left\{y_{n}\right\}$ of $\left\{x_{n}\right\}$ such that $\left\{\frac{1}{n} \sum_{k=1}^{n} T\left|y_{k}\right| ; \quad n \in \mathbb{N}\right\}$ is L-weakly compact subset. Since

$$
\left|\frac{1}{n} \sum_{k=1}^{n} S y_{k}\right| \leq \frac{1}{n} \sum_{k=1}^{n} T\left|y_{k}\right|,
$$

it follows that $\left\{\frac{1}{n} \sum_{k=1}^{n} S y_{k} ; \quad n \in \mathbb{N}\right\}$ is L-weakly compact.

In what follows:

$L(X, F)$ will denote the space of all operators from $X$ into $F$,

$S B S(X, F)$ will denote the space of all strong Banach-Saks operators from $X$ into $F$. 
Theorem 2.13. The set of all strong Banach-Saks operators from a Banach space $X$ to a Banach lattice $F$ is a closed vector subspace of $L(X, F)$.

Proof. Clearly, the collection $S B S(X, F)$ of all strong Banach-Saks operators from $X$ to $F$ is a vector subspace of $L(X, F)$.

Now, let $\left(T_{n}\right)_{n}$ be a sequence of strong Banach-Saks operators such that $\left\|T_{n}-T\right\| \rightarrow 0$ in $L(X, F)$. Let $\epsilon>0$, there exists $N_{0} \in \mathbb{N}$ such that $n \geq N_{0}$, implies $\left\|T_{n}-T\right\|<\frac{\epsilon}{2}$. Let $\left(x_{n}\right)$ be a bounded sequence in $X$, by passing to a subsequence, we can assume without loss of generality that $\left\{\frac{1}{n} \sum_{k=1}^{n} T_{N_{0}} x_{k} ; \quad n \in \mathbb{N}\right\}$ is L-weakly compact. The inequality

$$
\left|T\left(\frac{1}{n} \sum_{k=1}^{n} x_{k}\right)\right| \leq\left|\left(T-T_{N_{0}}\right)\left(\frac{1}{n} \sum_{k=1}^{n} x_{k}\right)\right|+\left|T_{N_{0}}\left(\frac{1}{n} \sum_{k=1}^{n} x_{k}\right)\right|,
$$

guarantees that $\left\{\frac{1}{n} \sum_{k=1}^{n} T x_{k} ; \quad n \in \mathbb{N}\right\}$ is L-weakly compact.

Proposition 2.14. Let $E=\prod_{i=1}^{n} E_{i}$ be the direct sum of Banach lattices, and let $A_{i j}: E_{j} \longrightarrow E_{i}$ be a strong Banach-Saks operator for all $1 \leq i, j \leq n$. Then the matrix operator $T: E \longrightarrow E$ defined by

$$
T=\left(\begin{array}{cccc}
A_{1,1} & A_{1,2} & \cdots & A_{1, n} \\
A_{2,1} & A_{2,2} & \cdots & A_{2, n} \\
\vdots & \vdots & \ddots & \vdots \\
A_{n, 1} & A_{n, 2} & \cdots & A_{n, n}
\end{array}\right)
$$

is strong Banach-Saks.

Proof. Let $\left\{X_{k}=\left(x_{k}^{1}, x_{k}^{2}, \ldots, x_{k}^{n}\right), k \in \mathbb{N}\right\}$ be a norm bounded sequence in $E$. Then $\left(x_{k}^{i}\right)_{k}$ is bounded in $E_{i}$ for each $1 \leq i \leq n$. Since $A_{i j}$ is strong Banach-Saks, then, by passing to a subsequence, we can suppose that $\left\{\sum_{j=1}^{n} \frac{1}{k} \sum_{l=1}^{k} A_{1 j} x_{l}^{j}, \quad n \in \mathbb{N}\right\}$ is L-weakly compact subset of $E_{i}$ for all $1 \leq i \leq n$. Then

$$
\frac{1}{k} \sum_{l=1}^{k} T X_{l}=\left(\begin{array}{c}
\sum_{j=1}^{n} \frac{1}{k} \sum_{l=1}^{k} A_{1 j} x_{l}^{j} \\
\sum_{j=1}^{n} \frac{1}{k} \sum_{l=1}^{k} A_{2 j} x_{l}^{j} \\
\vdots \\
\sum_{j=1}^{n} \frac{1}{k} \sum_{l=1}^{k} A_{n j} x_{l}^{j}
\end{array}\right)
$$

is L-weakly compact subset of $E$.

\section{ORder CONtinuous BanaCh lattices}

The next Theorem gives a characterization of Banach lattices with order continuous norms.

Theorem 3.1. For a Banach lattice $E$ the following statements are equivalent.

(1) $E$ is order continuous.

(2) $E$ is Dedekind $\sigma$-complete and $x_{n} \downarrow 0$ implies $\frac{1}{n} \sum_{l=1}^{n} x_{l} \rightarrow 0$.

(3) For every $x \in E_{+}$, the order interval $[-x, x]$ is Banach-Saks.

Proof. (1) $\Longrightarrow(2)$ If $x_{n} \downarrow 0$, then $\left\|x_{n}\right\| \longrightarrow 0$. Thus, $\frac{1}{n} \sum_{l=1}^{n} x_{l} \rightarrow 0$.

$(2) \Longrightarrow(1)$ Let $x_{n} \downarrow 0$, then $\frac{1}{n} \sum_{l=1}^{n} x_{l} \rightarrow 0$. In particular, from Lemma 2.6 it follows that $\overline{E_{A}}$ is order continuous, where $A=\left\{\frac{1}{n} \sum_{l=1}^{n} x_{l}, \quad n \in \mathbb{N}\right\}$. Since $x_{n} \in E_{A}$ for all $n \in \mathbb{N}$, then from Theorem 4.9 in [1] it should be clear that $\left\|x_{n}\right\| \longrightarrow 0$.

$(1) \Longrightarrow(3)$ Let $\left(x_{n}\right)_{n}$ be a sequence in $E$ satisfying $0 \leq x_{n} \leq x$ for all $n$ and some $x \in E_{+}$. Since $E$ is order continuous, it follows from Theorem 4.9 in [1] that [0, $x$ ] is weakly compact. By passing to a subsequence, we can assume that $x_{n} \stackrel{\sigma\left(E, E^{\prime}\right)}{\longrightarrow} y$ 
for some $y \in E$. Then $x_{n} \stackrel{\sigma\left(L_{1}(\mu), L_{\infty}(\mu)\right)}{\longrightarrow} y$ for some probability measure $\mu$. Thus, by Theorem 2.5 there exist a subsequence $\left(y_{n}\right)$ of $\left(x_{n}\right)$ such that $\frac{1}{n} \sum_{k=1}^{n} y_{k} \stackrel{\|\cdot\|_{1}}{\longrightarrow} y$. On the other hand, since $E$ is order continuous and $0 \leq \frac{1}{n} \sum_{k=1}^{n} y_{k} \leq x$ for all $n$, we see that $A=\left\{\frac{1}{n} \sum_{l=1}^{n} y_{l}, \quad n \in \mathbb{N}\right\}$ is L-weakly compact subset of $E$ ( see Theorem 4.14 in [1]). According to Lemma 2.4, we have $\frac{1}{n} \sum_{k=1}^{n} y_{k}$ converges to $y$ in $E$.

$(3) \Longrightarrow(1)$ The implication follows from Proposition 2.3 in [11].

\section{REFERENCES}

[1] C. D. Aliprantis and O. Burkinshaw, Positive Operators, springer, Dordrecht, (2006).

[2] A. Baernstein II, On reflexivity and summability, Studia Math, 42 (1972) 91-94.

[3] S. Banach and S. Saks, Sur la convergence forte dans les champs $L_{p}$. Studia Math. 2 (1930), 51-57.

[4] B. Beauzamy, Propriété de Banach-Saks, ibid. 66 (1980), 227-235.

[5] K. Bouras, D. Lhaimer and M. Moussa, On the class of almost L-weakly and almost M-weakly compact operators. Positivity 22, 1433-1443 (2018).

[6] A. Brunel and L. Sucheston, On J-convexity and some ergodic super-properties of Banach spaces, Proc. Amer. Math. Soc . 204 (1975), 79-90.

[7] R. Larsen, Functional analysis: An introduction, M. Dekker (1973).

[8] P. Meyer-Nieberg, Uber Klassen Schwach Kompakter Operatoren in Banachverbanden. Math. Z. $138,145-159$ (1974)

[9] P. Meyer-Nieberg, Banach lattices, Springer-Verlag, Berlin, Heidelberg, New York, (1991).

[10] T. Nishiura, D. Waterman, Reflexivity and summability, Studia Math. 23 (1963), 53-57.

[11] J. Lopez-Abad, C. Ruiz and P. Tradacete, The convex hull of a Banach-Saks set, J. Funct. Anal. (2013), 266(4), 2251-2280.

[12] P. Tradacete, Factorization and domination properties of operators on Banach Latices, Phd thesis, Universidad Complutense de Madrid (2010).

[13] L. Weis, Banach lattices with the subsequence splitting property, Proc. Am. Math. Soc. 105, 87-96, (1989).

[14] J. Lindenstrauss and L. Tzafriri, Classical Banach spaces II. Function Spaces, Springer-Verlag, (1979).

[15] M. Fabian, P. Habala, P. Hajek, V. Montesinos, and V. Zizler, Banach space theory: basis for linear and nonlinear analysis, Springer-Verlag, New York, 2011.

[16] W. Szlenk, Sur les suites faiblement convergentes dans l'espace l, Studia Math. 25 (1965), 337-341.

Mohamed HAJJ: medhajji.issatkasserine@gmail.com

Department of Mathematics and Computer Science, Issat Kasserine, BP 471, Kasserine, 1200, Tunisia

Received 15/04/2020; Revised 22/08/2020 